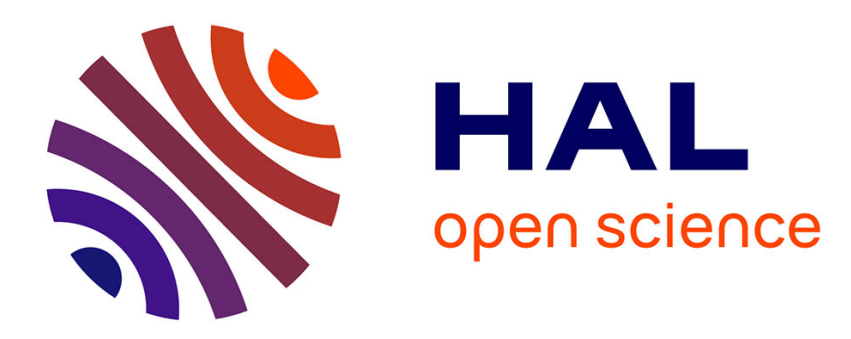

\title{
Incestualité et identification projective dans un cas d'infanticide
}

Florian Houssier, Estelle Louët

\section{To cite this version:}

Florian Houssier, Estelle Louët. Incestualité et identification projective dans un cas d'infanticide.

Cahiers de psychologie clinique , 2016, 47, pp.187 - 187. 10.3917/cpc.047.0187 . hal-01423197

\section{HAL Id: hal-01423197 \\ https://hal.science/hal-01423197}

Submitted on 28 Dec 2016

HAL is a multi-disciplinary open access archive for the deposit and dissemination of scientific research documents, whether they are published or not. The documents may come from teaching and research institutions in France or abroad, or from public or private research centers.
L'archive ouverte pluridisciplinaire HAL, est destinée au dépôt et à la diffusion de documents scientifiques de niveau recherche, publiés ou non, émanant des établissements d'enseignement et de recherche français ou étrangers, des laboratoires publics ou privés. 
Incestualité et identification projective dans un cas d'infanticide

Florian Houssier $^{1}$, E. Louët ${ }^{2}$

Résumé : A partir du suivi en milieu judicaire d'un homme souffrant de paranoïa, nous investiguons certains aspects dynamiques de son acte infanticide. Pour cela, nous nous appuyons notamment sur les concepts d'incestualité et d'identification projective afin de repérer les ressorts intrapsychiques d'un tel passage à l'acte et comprendre le fonctionnement psychique familial ainsi que la nature du lien entre ce père et son fils adolescent. Par rebond, la dimension psychopathologique de ces liens ouvre sur ce qui caractérise une parentalité non pathologique.

Mots clés : Infanticide - Paranoïa - Incestualité - Identification projective - Parentalité

Summary: We intend to investigate some dynamic aspects of an infanticide act from the judicial follow of a paranoid man. Thus we'll use concepts such as incestuality and projective identification in order to understand the inconscious meanings of the infanticide act. The singularity of the familial psychic functionning questions the pathological link between father and son, which are investigated such as the pathological parenthood.

Key words : Infanticide - Paranoia - Incestuality - Projective identification - Parenthood

\section{Introduction}

Lorsqu'un meurtre intrafamilial est agi, il est régulièrement associé à un climat incestueux et/ou incestuel dans la famille (Houssier, 2013). Dans l'opposition usuelle entre inceste agi et fantasme œdipien, on peut intercaler la notion d'incestualité (Racamier, 1995), plus proche d'un inceste non agi que de l'accès aux constructions imaginaires œdipiennes impliquant

\footnotetext{
${ }^{1}$ Psychologue, Psychanalyste, Président du Collège International de l'Adolescence (CILA), Pr de Psychologie clinique et Psychopathologie, Unité Transversale de Recherches : Psychogenèse et Psychopathologie (UTRPP), Université Paris 13, Villetaneuse, Sorbonne Paris Cité.

${ }^{2}$ Psychologue, Psychothérapeute, Maitre de conférences dans le laboratoire de psychologie clinique de psychopathologie et de psychanalyse (PCPP EA 4056), Institut de psychologie, Université Paris Descartes
} 
l'existence d'un tiers. Ce fonctionnement familial tend à chosifier, par un désir d'emprise, la psyché comme le corps de l'enfant; ce dernier est utilisé comme un objet narcissique manipulable, niant l'élaboration de la reconnaissance et de la différence entre les besoins de l'enfant et de l'adulte.

Pour éclairer sous un angle psychanalytique un cas de paranoïa aboutissant à un crime infanticide, nous nous appuyons essentiellement sur deux concepts dynamiques, l'incestualité et l'identification projective pour proposer des hypothèses dynamiques sur le fonctionnement psychique familial comme sur le lien fantasmatique entre un père et son fils adolescent.

Si l'identification projective est un concept désormais connu dans le champ psychanalytique, il nous parait opportun de revenir sur ce qu'on entend par incestualité (ou l'incestuel), terme parfois utilisé sans nuances. L'incestualité est une notion introduite par le psychiatre et psychanalyste français P. C. Racamier, à partir d'observations de patients psychotiques (Racamier, 1980); il a étendu l'usage de ce concept à d'autres configurations psychiques, non psychotiques mais ayant en commun des défauts de symbolisation (Racamier, 1989). La relation incestuelle est caractérisée par le même type de liens étroits qu'uniraient deux personnes vivant une relation incestueuse, sans cependant réaliser celle-ci. Elle se présente ainsi comme un «fantasme agi », scénarisé, mais amputé des propriétés ou des fonctions du fantasme - dans leur fonction de vectorisation du désir et de conflictualisation psychique ; quant à la banalisation entourant l'évocation de telles relations, elle est le plus souvent empreinte de déni.

Pour Racamier, ce déni porte sur l'interdit de l'inceste, ce qui permet que l'irruption du désir sexuel n'altère en rien l'union narcissique fondamentale. L'usage du terme a été étendu pour caractériser certains fonctionnements familiaux où règne un «climat incestuel » marqué par le manque de limites, les confusions des identités, les intrusions, ou encore la paradoxalité induite dans les échanges.

Si Racamier (1995) ne parle pas d'identification projective, il s'en approche en proposant le terme «d'engrènement » pour expliciter le fonctionnement incestuel familial : l'engrènement est défini comme un processus mettant une psyché en prise directe avec celle d'un autre sans qu'une médiation - fantasmatique, relationnelle ou culturelle - puisse intervenir. La particularité de l'engrènement est que ce processus n'est pas seulement d'essence psychique ; c'est un circuit interactif anti-pensée relevant de l'articulation agir-faire agir. Agir-faire agir s'articule donc avec des fantasmes anti-fantasmes (Idem), sur fond d'indifférenciation entre sujet et objet. Les situations incestuelles, qui s'établissent sur fond de confusion ou de déni 
des registres symboliques, sont par conséquent porteuses de passage à l'acte potentiel, comme l'illustre le cas de Patrick: cette situation clinique montre de façon aussi exemplaire que tragique les liens étroits entre incestualité, passage à l'acte et parentalité en souffrance sur fond de déni d'altérité.

C'est dans la clinique du milieu judiciaire, en particulier dans le contexte des suivis socioéducatifs pré-sentenciels en milieu ouvert, que ces problématiques se retrouvent le plus fréquemment, c'est dans ce cadre que Patrick a été rencontré par l'un d'entre nous (Houssier, 2007).

\section{Mon enfant est à moi}

Patrick est âgé de 37 ans lorsqu'il est placé sous contrôle judiciaire - pendant un an - pour coups et blessures volontaires sur son fils aîné de 15 ans, alors qu'il était sous l'emprise de l'alcool. Treize ans auparavant, il a déjà été jugé pour coups et blessures avec arme, dans le contexte d'une bagarre entre frères : il a six frères et une sœur, il est le troisième ; il a quatre fils. Son père a disparu lors d'un accident de la route alors que Patrick avait 17 ans. Je rencontre donc Patrick pour des entretiens bi-mensuels, ce dernier ayant comme obligation judiciaire, avant son passage en jugement, de venir à ces rendez-vous qui ont lieu dans les locaux associatifs d'un tribunal de grande instance.

\section{$\underline{\text { Lâcher les chiens }}$}

La relation avec Patrick est d'emblée infiltrée par une rudesse dans le contact, sans filtre ni retenue de sa part. Dès le premier entretien, il fait part de sa méfiance envers le «système judiciaire » tout en acceptant sans difficulté apparente l'ouverture de cet espace de parole malgré le contexte judiciarisé. La colère et le ressentiment sont les premiers affects qu'il nous donne à entendre, tout en nous laissant penser l'importance du réglage de la distance avec lui. Spontanément, il évoque ses relations actuelles tourmentées avec son fils aîné, présenté comme un mauvais garçon qui tente de s'extraire de l'influence paternelle en quittant la maison pour aller voir ses amis ou fumer des cigarettes. Alors que Patrick souffre d'alcoolisme depuis son récent licenciement d'une entreprise où il était maçon, il craint que son fils ne devienne un drogué, qu'on dise qu'il a été mal éduqué et que cela lui retombe dessus. Car son sentiment est que les choses se font toujours à son détriment. «C'est comme 
mes frères, dès que mon père est mort, ils se sont mis à faire n'importe quoi, des délits. Ils n'avaient plus la peur du père », souligne-t-il pour rendre compte de son désir que tout le monde lui obéisse, à lui, dans sa famille. Sa femme tente de s'interposer entre lui et son fils, ce qu'il réprouve. De même, dans la relation avec nous, nous sommes régulièrement sollicités par l'image suivante : avec Patrick, nous avons l'impression de régulièrement « marcher sur des œufs ». Dans la dynamique transférentielle, proposer un discours contradictoire aux propos de Patrick ne paraît pas réalisable tant le lien ne peut exister qu'à condition d'accepter d'être un objet partiel, une oreille. La conflictualisation du lien ajouterait ainsi un nouveau persécuteur, tandis qu'entendre sans porter de jugement dans un contexte judiciarisé ouvre sur la possibilité d'entendre là où le sujet souffre et, au mieux, sur la réduction potentielle de certaines zones de clivage grâce au lien de confiance qui s'est instauré progressivement.

Patrick poursuit : «Avant, c'était deux-péccable, tout marchait comme il faut », dit-il pour évoquer la période où son fils aîné était encore un enfant. Mais ce qui aurait pu être un jeu sur les mots dont la polysémie ouvrirait sur un autre sens, se referme dans une voie à sens unique. Si le terme «impeccable » désigne l'absence de défaut, renvoie à l'incapacité à pêcher, à commettre une erreur, le néologisme deux-peccable englobe inexorablement père et fils dans l'univocité des liens fondés sur le régime de la terreur.

Le père le frappait sans mot dire, tandis que la mère punissait par des contraintes. Lors de cet accompagnement psychologique en milieu judiciaire aux effets thérapeutiques potentiels (Houssier, 2007), le seul moment au cours duquel Patrick s'effondre en sanglots se situe au moment où, à la faveur d'un transfert plutôt positif qui s'est désormais installé dans la relation, il évoque la violence des humiliations et des maltraitances paternelles envers lui : «Je le voyais bien, il traitait mieux son chien que moi », dit-il en pleurant. «J'aimais bien ce chien, moi aussi », ajoute-t-il alors comme identifié au chien recevant des gestes de tendresse $\mathrm{du}$ père, au moment où mon écoute et le développement progressif d'un lien de confiance semblent être vécus comme une forme d'attention tendre, dans un milieu judiciaire au potentiel persécutant. Dans le transfert, alors que certains occupent la place d'objet persécuteurs (les magistrats, le psychiatre expert), nous sommes plutôt situés dans le transfert comme une figure paternelle tendre, compréhensive, à la façon d'un père qui caresse son chien et dans le sens d'un transfert homosexuel plus tempéré que passionnel.

Malgré ce qu'il en relate, le lien à son père ne fait pas l'objet de critiques; il est même valorisé car il s'appuie sur des valeurs telles que «la politesse et la propreté »; que l'évocation de telles qualités chez un père s'accompagne d'un langage grossier aux 
connotations clairement anales, dans la bouche de Patrick ne fait ici l'objet d'aucune contradiction, d'aucun conflit. Le clivage qui divise la politesse et la grossièreté dans le discours de Patrick, nous parle d'un univers identificatoire empreint d'idéalisation de figures parentales toutes puissantes.

Au fil de ces entretiens, il fait le récit de ses revendications notamment auprès du maire de sa commune. Sa quérulence, sa méfiance et sa rigidité se déploient maintenant dans deux situations précises.

La première concerne le psychiatre expert qu'il a rencontré à la demande du juge d'instruction. Dans ses conclusions, celui-ci a mis en avant l'alcool pour expliquer les violences intrafamiliales, le psychiatre a insisté sur la nécessité d'une désintoxication, sans référence à la problématique paranoïaque de Patrick. Sa «réadaptation» est donc considérée comme tributaire dans une large mesure de la prise en charge de sa problématique alcoolique. Mais ce n'est pas ce qui dérange Patrick lorsqu'il me parle de ce psychiatre : il a été choqué que celuici dise à sa femme de mettre un stérilet pour qu'ils n'aient plus d'enfant, et d'avoir été traité à plusieurs reprises de «tête de cochon». Ces phrases, que le psychiatre expert aurait prononcées, apparaissent comme autant de blessures narcissiques persécutantes qui l'obsèdent encore plusieurs mois après cette unique rencontre ; l'insistance avec laquelle Patrick évoque l'épisode et la quérulence qui accompagne ses propos, interrogent la composante homosexuelle d'une telle fixation, dont la coloration haineuse rappelle avec insistance le lien à son propre père.

Un nouveau déplacement de ses affects intervient sans crier gare lorsqu'un nouvel objet persécuteur apparaît, sous la forme de la juge pour enfant.

La relation avec celle-ci crée chez lui une tension insupportable dès le premier entretien avec elle; saisie pour le motif de mineur en danger, elle lui a indiqué qu'elle envisageait de lui retirer la garde de son fils. Pendant plusieurs entretiens, une idée l'obsède : elle l'a blessé en le traitant de mauvais père. Lorsqu'il est question de lui retirer la garde de son fils, il explose : si les gendarmes viennent, il enverra les chiens puis il les recevra à coups de fusils, éructe-t-il. «Lâcher les chiens », la formule fait apparaître l'autre versant identificatoire attaché à cet objet si investi par le père, son chien : cette expression renvoie à la projection d'une violence déliée, sans frein. Comme les enfants, les chiens sont ici aussi bien dépositaires de la haine que du besoin de tendresse. 
L'image qui l'envahit désormais est celle des gendarmes surgissant chez lui ; cette intrusion dans son espace intime est imaginée comme un viol et une dépossession du pouvoir d'emprise, répétés au fil des générations dans les relations père-fils.

La double dimension incestueuse - dans le corps à corps des coups - et incestuelle, par l'indifférenciation des psychés, est centrale: sa famille est le lieu de son intimité, elle constitue à ce titre un corps où les différents membres sont indissociables, sous peine d'effondrement. A la différenciation potentielle s'oppose le «deux-péccable » totalisant, dans une unité homosexuelle indistincte. Lorsqu'il explose contre la juge pour enfants et ses délégués, le sentiment est qu'il peut réellement faire ce qu'il dit; car ce qu'il évoque ne constitue pas un scénario épaissi par la tessiture du fantasme, mais bien plutôt une possibilité d'extériorisation, de mise en acte, de sa toute-puissance. Il ajoute qu'il est prêt à faire de la prison, qu'il s'en fiche. Grâce à la qualité du transfert, nous pouvons le renvoyer alors à luimême, à la difficulté de cette situation pour lui, à ce que serait la prison pour lui, paroles lui permettant d'être davantage en contact avec ses affects. Entretien après entretien, l'espace de parole qui lui est offert lui permet d'exprimer sa folie et son déni de toute loi, ce qui semble contenir un potentiel passage à l'acte. Celui-ci est comme anticipé et évacué par des décharges verbales, sans rétorsion ni jugement en retour; la co-création d'un espace psychique en milieu judiciaire n'est possible qu'à condition de ne pas se sentir responsable des agissements éventuels dans la réalité, de se dégager intérieurement d'un collage potentiel au réel d'un acte, au risque de clivages aux effets destructeurs potentiels.

Le jugement est rendu, Patrick est condamné à une peine de sursis mais le fils reste finalement chez ses parents.

Deux ans plus tard, en croisant dans les couloirs du tribunal un juge d'instruction, celui-ci me reconnaît et me dit : «C'est bien vous qui avez suivi $\mathrm{M}$. Untel il y a deux ans ? ». Lorsque j'acquiesce, elle me dit qu'elle s'occupe d'un nouveau dossier concernant Patrick : il a tué son fils aîné et sa femme avec son fusil. Son fils avait 17 ans, l'âge où le père de Patrick est mort et où il est parti de chez lui sans rien dire à sa mère. Lorsque, encore sidéré par cette annonce inattendue, je dis qu'il s'agit d'un sujet souffrant de paranoïa, le magistrat me répond qu'elle a ordonné une nouvelle expertise, et que celle-ci conclut à l'absence de toute maladie mentale. S'il ne peut être question de rabattre la complexité d'une telle situation sur les aléas des rouages institutionnels ou expertaux, il est difficile de ne pas penser aux défauts de contenance du filet judiciaire et social qui, trop souvent, caractérisent des prises en charge 
trouées par la discontinuité des accompagnements, qu'ils soient sociaux-judiciaires ou psychologiques (Chagnon, Houssier, 2014).

\section{La quête d'un parent pur ?}

\section{L'identification projective pathologique, un pousse au crime}

Au-delà des failles dans le tissage des soins dans le cadre judicaire, quel sens peut on donner dans l'après-coup à ce passage à l'acte infanticide ? Sans rabattre la problématique d'un enfant sur les liens qu'il entretient avec les membres de sa famille ou sur les événements qui ont jalonné son développement, notamment traumatiques, on peut penser que le fils de Patrick a été l'objet-dépôt du narcissisme paternel. Le caractère insupportable de l'écart créé entre l'attitude de son fils et les idéaux attachés au père violent de Patrick représente un premier aspect, impliquant trois générations ; la honte liée au regard intériorisé du père de Patrick est un pousse-au-crime : tuer son fils c'est supprimer la source d'un sentiment de déchéance et d'impuissance face aux mouvements d'indépendance de son fils qu'il vit comme autant de provocations. Sous un autre angle, complémentaire, la haine niée de Patrick pour son propre père est retournée contre son fils, objet et réceptacle de l'identification projective de son père. Ce qui a été refusé - la représentation de désir de mort envers son père - ressurgit pour être en quelque sorte pris en charge par l'infanticide, via le retour agi du désir clivé. L'identification projective est une force agissante dans le sens où Patrick s'identifie projectivement à celui qui l'a maltraité, son père, pour faire subir à son fils cette violence.

La dimension traumatique des violences génère une transmission avec peu de transformations, si ce n'est dans l'intensité de ce qui fait retour via le passage à l'acte. Cette violence est ainsi expulsée en la projetant dans un objet (le fils) imaginé sous contrôle : l'identification projective intervient également pour insuffler ce fantasme omnipotent, ici conscient, véhiculant l'idée que pénétrer l'objet psychiquement par des ordres et autres injonctions tyranniques a pour visée de le contrôler de l'intérieur, d'en prendre possession sans obstacle (Ciccone, 1999). Toute mise en défaut de cette illusion, par l'existence d'un désir autre et opposé, attaque l'édifice narcissique de Patrick, renvoyant à l'idée de tuer pour survivre.

Dans la situation de Patrick, sa femme est vécue comme un obstacle persécutant faisant barrage au maintien de l'omnipotence, et non comme un médiateur dans le lien parentsenfant; son meurtre vient représenter un fantasme d'engendrement : le père doit rester à l'origine de toute chose, y compris du droit de vie et de mort. Dans ce contexte, 
l'identification projective prend une place centrale, si on le comprend comme une façon de faire vivre inconsciemment un fantasme d'immortalité et de toute-puissance à travers ce qui est projeté sur l'enfant. Le meurtre du fils comme de sa mère laisse entrevoir que ceux-ci ont été perçus comme un couple incestueux, mais aussi comme ceux qui voulaient se soustraire à la loi paranoïaque du père de Patrick, introjecté sous la forme d'un surmoi préoedipien cruel. Ce double meurtre peut être compris comme une façon de défendre l'image de son père et le lien indéfectible qui unit père et fils. Il représente également le meurtre d'un couple d'un couple parental incluant un matricide ; car ce n'est pas tant sa femme que la mère de son fils qu'il a tuée.

Par le meurtre, Patrick défend une image de parent pur, l'idéalisation barrant toute élaboration de la relation préœdipienne entre parent et enfant. L'idéalisation est un cache-haine qui protège la figure parentale primaire et s'oppose à toute secondarisation des liens aux objets primitifs.

\section{$\underline{\text { L'arbitraire des lois intimes dans la parentalité }}$}

En creux, la problématique de Patrick illustre un des temps indissociables de la parentalité : au début de la vie, le bébé est une part narcissique de soi, peu différenciée dans le bain narcissique parents-enfant. Cette part de soi attribuée à l'enfant - «Comme il me ressemble », semble dire chaque parent - constitue un investissement indispensable à la croissance psychique de l'enfant: pas de support identificatoire possible pour l'enfant sans investissement narcissique massif de «His Majesty the Baby (Freud, 1914). L'enfant est bien un jouet érotique, dans l'amour aliénant comme dans la haine de la différence ; cette différence inter-objectale constitue un écart entre les deux subjectivités, parentale et infantile, qui se révèle insupportable pour Patrick.

Pour Patrick, le sentiment d'appartenance de son enfant devenu adolescent laisse penser qu'il n'a jamais pu considérer son fils comme un sujet séparé et différencié de lui, développant ses propres besoins, sa propre vie psychique. Au moment de l'adolescence, les enjeux de différenciation (Houssier, 2012) entrent en collusion avec ce fantasme omnipotent d'appartenance qui pourrait être résumé de la sorte : «Nulle loi (réelle ou symbolique) ne peut m'enlever mon enfant ni lui donner une indépendance vis-à-vis de mes désirs ». On retrouve dans cette loi personnelle non partageable le bain narcissique qui entoure les débuts de la vie de l'enfant, donnant lieu à des fantasmes omnipotents chez l'enfant, qui imagine 
avoir créé le sein maternel et l'environnement dans son ensemble (Winnicott, 1971). Chez le parent, comme en écho, le fantasme omnipotent peut prendre la forme d'une assimilation dévoratrice, en lieu et place d'une capacité d'empathie et de sollicitude envers l'enfant. Les meurtres, notamment familiaux, provoquent souvent des réactions violentes dans le public, certaines personnes réclamant le rétablissement de la peine de mort pour le meurtrier. Sans le savoir, ces personnes sont identifiées non pas à la réparation de la victime, comme ils l'imaginent, mais plutôt à la toute-puissance du parent qui dirait ici, à travers son crime : «Nul d'autre que moi a droit de vie ou de mort sur mon enfant ». Ce fantasme est voisin, dans les situations d'inceste, de l'idée : «Nul autre que moi n'a la droit d'avoir des relations sensuelles ou sexuelles avec mon enfant », rejetant toute relation extra-familiale.

Ce type de fantasme narcissique fait de l'enfant un objet pris dans une possessivité indifférenciatrice, prisonnier d'un langage de la passion excluant toute intervention tierce. Plutôt le meurtre que l'acceptation-intériorisation de la perte, semble dire Patrick à travers son passage à l'acte ; tout en se vengeant des blessures narcissiques réactualisées, il empêche les objets de son amour - sa femme et le fils adolescent qui font l'objet de sa fixation persécutive - d'appartenir à quelqu'un d'autre que lui.

Le langage de la passion scelle ici le destin de la mère et du fils, qui seront tués plutôt que perdus. Le meurtre, en toute possessivité mélancolique, signe le radical refus des effets de l'objet sur le sujet et en ce sens interroge les effets d'une impossible confrontation à la position passive éprouvée au sein même des scènes originaires et de toute situation d'exclusion ou d'abandon. (Louët \& Chabert, 2011). Cette forme de tyrannie que constitue le meurtre apparaît comme le paravent d'une angoisse de perte déniée et clivée, mais également comme une forme de recomposition délirante des origines : tuer revient à refonder des origines familiales par la mise à mort, le fantasme de scène primitive étant alors dominé par une scène dans laquelle l'union des corps est meurtrière. Comme le souligne Freud (1895), la paranoïa est une combinaison de mégalomanie et de création de mythes quant aux origines de l'enfant.

\section{Conclusion : de la reconnaissance du sujet dans la parentalité}

Dans les enjeux psychiques de la parentalité, la reconnaissance de l'enfant comme sujet différencié s'inscrit dans le contexte d'une relation empathique où la tendresse du parent est au premier plan, les désirs sexuels étant eux l'objet du refoulement. Lorsqu'elle est inversée, 
cette articulation psychique « organise » les liens familiaux : cruauté et sexualité narcissique, meurtre de la capacité à élaborer les fantasmes incestueux en désirs oedipiens, appropriation narcissique du corps de l'enfant comme un objet sexuel à soi prédominent sur la tendresse, qui implique l'altérité dans le sens de l'accès à l'ambivalence et au stade de la sollicitude (Winnicott, 1971).

Pour un parent, la rigidité et la fixité de l'idéalisation de son enfant s'articulent avec le déni de sa subjectivité; un parent tout-puissant est animé par un fantasme d'appropriation cannibalique par assimilation, possession et indifférenciation parent-enfant. De cette indifférenciation des psychés entre parent et enfant dérivent une confusion qui ressort fréquemment dans les propos de Patrick, sur fond de déni de la différence entre soi et l'autre. Confondre et nier sont ainsi les deux enjeux antagonistes au mouvement de reconnaissance de l'enfant en tant que sujet. Le meurtre est pris dans les enjeux de différenciation, toute tentative d'exister pour soi étant vécu comme une agression pour le parent, provoquant une retaliation paranoïde de type «Eil pour œil, dent pour dent». Le clivage bon/mauvais enfant rappelle celui qui marque les premiers temps de la vie psychique du nourrisson concernant le bon et le mauvais sein (Klein, 1968) ; ce clivage résulte du dépôt des identifications projectives parentales qui font retour, notamment à l'adolescence. La situation de Patrick montre à quel point il est nécessaire d'être deux pour se séparer, et pour un parent, laisser se développer les mouvements de différenciation et de personnalisation; lorsque ceux-ci, notamment au moment de l'adolescence, sont vécus comme un écart insupportable avec l'exigence idéalisante transgénérationnelle, il est question de supprimer ce qui revient sous la forme d'une souffrance agonistique.

\section{$\underline{\text { Bibliographie }}$}

Chagnon J.-Y., Houssier F. (2014). «Le socius et la position de la justice face à l'enfant délinquant », in Cohen de Lara A., Danon-Boileau L., (dir.), La destructivité chez l'enfant, Les Monographies de la Société Psychanalytique de Paris, Collection Débats de Psychanalyse, Paris, PUF, p. 75-97.

Ciccone A. (1999). La transmission psychique inconsciente, Paris, Dunod.

Freud S. (1895). La naissance de la psychanalyse, Paris, PUF, 1973.

Freud S. (1914). « Pour introduire le narcissisme», in La vie sexuelle, Paris, PUF, 1973, p. 81105. 
Houssier F. (2007). « Les paradoxes du travail thérapeutique institué par décision de justice : interrogations sur le cadre interne à partir du contre-transfert». In R. Scelles, A. Aubert (Ed.), Dispositifs de soins au défi des détresses extrêmes, Ramontville Saint-Agne, Erès, p. 105-128. Houssier F. (2012). «Vœux parricides et fantasmes de dévoration. De la désidéalisation du père à l'adolescence », Psychiatrie de l'enfant, LV, 2, p. 563-579.

Houssier F. (2013), Meurtres dans la famille, Paris, Dunod.

Klein M. (1968). Essais de psychanalyse, Paris, Payot.

Louët E., Chabert C. (2011). «La mélancolie, un destin de la passivité », L'évolution psychiatrique, 76, 1, p. 31-42.

Racamier P.-C. (1980). Les schizophrènes, Paris, Payot.

Racamier P.-C. (1989). Antoedipe et ses destins, Paris, Apsygée.

Racamier P.-C. (1995). L'inceste et l'incestuel, Paris, Apsygée.

Winnicott D.W. (1971). L'enfant et sa famille, Paris, Payot. 